\title{
Structural and magnetic properties of CoO-Pt core-shell nanoparticles
}

\author{
Adriana Zeleňáková, ${ }^{1,}{ }^{*}$ Vladimir Zeleňák, ${ }^{2}$ Štefan Michalík, ${ }^{1,3}$ Jozef Kováč, ${ }^{4}$ and Mark W. Meisel ${ }^{1,5}$ \\ ${ }^{1}$ Department of Condensed Matter Physics, P. J. Šafárik University, Park Angelinum 9, 04154 Košice, Slovakia \\ ${ }^{2}$ Department of Inorganic Chemistry, P. J. Šafárik University, Moyzesova 11, 04154 Košice, Slovakia \\ ${ }^{3}$ Institute of Physics, Czech Academy of Sciences, Na Slovance 2, 18221 Prague, Czech Republic \\ ${ }^{4}$ Institute of Experimental Physics, Slovak Academy of Sciences, 04101 Koice, Slovakia \\ ${ }^{5}$ Department of Physics and NHMFL, University of Florida, Gainesville, Florida 32611-8440, USA
}

(Received 5 November 2013; revised manuscript received 13 February 2014; published 20 March 2014)

\begin{abstract}
Using microemulsion methods, CoO-Pt core-shell nanoparticles, with diameters of nominally $4 \mathrm{~nm}$, were synthesized and characterized by high-resolution transmission electron microscopy and a suite of $\mathrm{x}$-ray spectroscopies, including diffraction, absorption, absorption near-edge structure, and extended absorption fine structure, which confirmed the existence of $\mathrm{CoO}$ cores and pure Pt surface layers. Using a commercial magnetometer, the ac and dc magnetic properties were investigated over a range of temperature $(2 \mathrm{~K} \leqslant T \leqslant$ $300 \mathrm{~K})$, magnetic field $(\leqslant 50 \mathrm{kOe})$, and frequency $(\leqslant 1 \mathrm{kHz})$. The data indicate the presence of two different magnetic regimes whose onsets are identified by two maxima in the magnetic signals, with a narrow maximum centered at $6 \mathrm{~K}$ and a large one centered at $37 \mathrm{~K}$. The magnetic responses in these two regimes exhibit different frequency dependencies, where the maximum at high temperature follows a Vogel-Fulcher law, indicating a superparamagnetic blocking of interacting nanoparticle moments and the maximum at low temperature possesses a power-law response characteristic of a collective freezing of the nanoparticle moments in a superspin glass state. This co-existence of blocking and freezing behaviors is consistent with the nanoparticles possessing an antiferromagnetically ordered core, with an uncompensated magnetic moment, and a magnetically disordered interlayer between the $\mathrm{CoO}$ core and the Pt shell.
\end{abstract}

DOI: 10.1103/PhysRevB.89.104417

PACS number(s): 75.75.Fk, 75.50.Vv, 61.05.cj, 75.40.Gb

\section{INTRODUCTION}

Over the past few decades, cobalt based nanoparticles (NPs) have attracted a significant amount of research interest because of their applications in ferrofluids, electronic components, solar energy transformers, anodes for batteries, and chemical catalysts [1-3]. Such nanoparticles are one of the leading candidates for high density magnetic recording media, where the particles with small size, narrow size distribution, and controlled shape are required [4].

In addition, fine Co-based particles are model materials for fundamental investigations of a variety of magnetic phenomena, such as exchange spring and exchange bias in magnetically hard and soft phases of antiferromagnetic (AFM) and ferromagnetic (FM) interlayers, respectively $[5,6]$. Studies of the dynamics of the Co based nanoclusters showed interesting features such as pure superparamagnetic (SPM) relaxation [7-9], spin canting [10], and superspin glass (SSG) behavior $[6,11]$. More specifically, the SSG behavior is typically manifested in strongly interacting and dense nanoparticle systems showing spin-glass (SG) behavior. The evidence of SSG transition in fine-particle systems is strengthened by standard spin-glass fingerprints, namely the critical slowing down of the relaxation and the divergence of the nonlinear susceptibility at a finite glass transition temperature $T_{g}$ [11]. Finally, based on numerous studies, interparticle dipole-dipole interactions are known to increase the average blocking temperature and affect the height and distribution of the energy barriers [12].

\footnotetext{
*adriana.zelenakova@upjs.sk
}

All of these interesting magnetic phenomena in nanoparticles can be induced and influenced by a combination of particle sizes and surface effects [13-25]. Especially in small nanoparticles, the surface effects play an important role in the tuning of the magnetic behavior as the decreasing of particle size leads to an increasing fraction of atoms lying at or near the surface, where uncompensated surface spins can generate a net magnetic moment [26]. These interface effects are more pronounced in antiferromagnetic nanoparticles than ferromagnetic ones because of the lower magnetic moment of their cores. Anomalous magnetic properties arising from complicated surface effects have been the focus of experimental studies over the past few years by investigations involving antiferromagnetic nanoparticles of $\mathrm{NiO}[15,16,27,28]$, $\alpha-\mathrm{Fe}_{2} \mathrm{O}_{3}$ [29], $\mathrm{FeOOH} \cdot n \mathrm{H}_{2} \mathrm{O}$ [30], and $\mathrm{CoO}$ [31-33]. In fact, $\mathrm{CoO}$ is specifically germane to the present study, and its bulk form exhibits antiferromagnetic ordering at a Néel temperature $T_{\mathrm{N}}=298 \mathrm{~K}$. Above $T_{\mathrm{N}}, \mathrm{CoO}$ possesses a $\mathrm{NaCl}$ structure, whereas below $T_{\mathrm{N}}$, unstressed crystals experience tetragonal contractions along the cubic [100] directions, giving rise to domains [34-36]. Reducing the particle size to the nanoscopic scale reduces $T_{\mathrm{N}}$ and dramatically changes the magnetic properties [32].

Given the broad range of work reported, it is noteworthy that investigations involving antiferromagnetic $\mathrm{CoO}$ nanoparticles with Pt coatings has not been reported. In this paper, we describe the detailed structural and magnetic study of CoO-Pt core-shell nanoparticles with diameters near $4 \mathrm{~nm}$, where the choices of the Pt coating and the size of particles were driven by the desire to avoid agglomeration and to enhance potential biomedical applications. Due to the small size of the nanoparticles being studied, the detailed structure properties were investigated using the methods of $\mathrm{x}$-ray-absorption 
near-edge structure (XANES) and extended x-ray-absorption fine structure (EXAFS) giving information about the number and type of neighboring atoms, interatomic distances, and disorder. Theoretical simulations were performed to illustrate the effect of crystalline structure on the EXAFS pattern. Analysis of results of magnetic measurements (dc magnetization and ac susceptibility) showed a combination of superparamagnetic blocking and collective superspin glass freezing. The interpretations of the results indicate that the presence of $\mathrm{Pt}$ shell is the key factor in observed magnetic behavior. Specifically, the Pt shell, in combination with the small size of Co-based core, can polarize (or frustrate) the spins at the surface and in the near-surface regions of antiferromagnetic $\mathrm{CoO}$ nanoparticles. Such cooperation of two different processes with completely different spins dynamics in Co-based nanoparticles is an important finding of this work. Usually the existence of shell layers with different spin dynamics leads to an exchange bias effect. However, in the sample under study, no bias mechanism was observed, thereby indicating that the mechanism of spin-glass freezing that leads to the SSG state is mainly influenced by the strong dipolar magnetic interactions of superspins.

The presentation of our work starts by providing the details of the synthesis protocols used to fabricate the samples, and the subsequent characterization methods used to explore the chemical composistion and morphology. Next, the magnetic investigations and results are described, while an extensive discussion of the analysis of all of the data is given. Finally, the paper concludes with a summary of our findings.

\section{EXPERIMENTAL DETAILS}

\section{A. Sample preparation}

The CoO-Pt core-shell nanoparticles were synthesized using the reverse micelle concept [37-39], based on the dissolution of a cationic surfactant in an organic solvent and the formation of spherical reverse micelle aggregates. All chemicals were purchased from Aldrich and Sigma and used without further purification. The reverse micelle solutions were prepared using cetyltrimethylammonium bromide (CTAB) as the surfactant with octane as the oil phase, while 1-butanol was used as a cosurfactant helping to stabilize the micelle solutions. Water solutions of $\mathrm{CoCl}_{2}, \mathrm{NaBH}_{4}$, and $\mathrm{H}_{2} \mathrm{PtCl}_{6}$ were used for the formation of initial droplets in the reverse micelles, whose reaction led to the final nanoparticles. The size of the particles (water droplet size) was controlled by adjusting the water-to-surfactant molar ratio $\left[\mathrm{H}_{2} \mathrm{O} / \mathrm{CTAB}\right]=5$. The $\mathrm{Co}$ based particles formed the cores coated by a nonmagnetic Pt surface layer. This process prevents the agglomeration of particles and is effective in preparing particles with metal cores and different shells.

\section{B. Characterization}

High-resolution transmission electron microscopy (HRTEM) and electron-diffraction analysis were performed with a JEOL JEM 3010 transmission electron microscope operated at $300 \mathrm{kV}$ ( $\mathrm{LaB}_{6}$ cathode). Copper grids coated with a holey carbon support film were used to prepare samples for the TEM studies. Powdered samples were dispersed in ethanol, and the suspension was treated in an ultrasonic bath for $10 \mathrm{~min}$.

The structure of the nanoparticles was investigated by means of the angular dispersive X-ray-diffraction (XRD) and $\mathrm{X}$-ray-absorption spectroscopy (XAS). The XRD measurements using high-energy photons were performed at the wiggler beamline BW5 at HASYLAB/DESY (Hamburg, Germany). The wavelength of the radiation was set to $0.12398 \AA$, which corresponds to the beam energy of $100 \mathrm{keV}$. Thin-walled $(20 \mu \mathrm{m})$ quartz capillaries having diameters of $2 \mathrm{~mm}$ were used for the XRD measurements. A powder sample was illuminated for $50 \mathrm{~s}$ with the well collimated beam having a cross section of $1 \times 1 \mathrm{~mm}^{2}$. $\mathrm{A} \mathrm{LaB}_{6}$ standard was used to calibrate the sample-to-detector distance along with the tilt of the imaging plate relative to the beam path and to determine the instrumental broadening. Diffraction patterns were collected in transmission mode using a MAR345 image plate detector. Two-dimensional XRD patterns were radially integrated using the FIT2D program [40].

The local atomic arrangement was investigated by $\mathrm{x}$ ray-absorption spectroscopy (XAS), which provides complementary information to XRD measurements though offering better chemical sensitivity. The XAS measurements were conducted at the bending magnet experimental stations $\mathrm{C}$ and $\mathrm{X} 1$ at HASYLAB/DESY. The fine oscillations of the linear absorption coefficient $\mu(E)$ were measured at the Co $K$ edge $(7709 \mathrm{eV})$ and at the Pt $L_{3}$ edge $(11564 \mathrm{eV})$ in transmission mode using a fixed exit double-crystal Si (111). In order to optimize the absorption signal, powder nanoparticles $(7.1 \mathrm{mg})$ were uniformly dispersed with cellulose powder (200 mg). Compressing the mixture using a hydraulic press yielded a sample pellet with diameter and height of 13 and $3 \mathrm{~mm}$, respectively. The pellet was placed behind the first ionization chamber and was illuminated by the incoming beam having the cross section $5 \times 1 \mathrm{~mm}^{2}$. The energy calibration was performed simultaneously with the sample measurement by putting the corresponding reference Co or Pt foil behind the second ionization chamber. An experimentally measured $\mathrm{X}$-ray-absorption cross section $\mu(E)$ was analyzed by the standard procedures of data reduction described elsewhere $[41,42]$ using the program VIPER $[41,43]$. First, the EXAFS signal $\tilde{\chi}(k)$ was extracted, weighted by $k^{2}$, and subsequently Fourier transformed (FT) into the real space of interatomic distances. Then the main peak of the FT- $\left|k^{2} \tilde{\chi}(k)\right|$ signal was separated by applying the so-called Hanning window function with the coefficient $A=0.01$. The resulting data were then inverse Fourier transformed back into $k$ space, and these filtered data only contain information about the atoms nearest to the absorbing ones (first coordination shell). Finally, fitting by an appropriate model yields structural information about the coordination number $N$, the interatomic distance $r$, and the Debye-Waller factor $\sigma$. The backscattering amplitude $F_{i}(k)$ and phase shift $\tilde{\chi}_{i}(k)$ functions, necessary for computation of the EXAFS signal from the model, were calculated using the FEFF6 code [44].

Magnetic measurements were performed on a commercial superconducting quantum interference device (SQUID) magnetometer (Quantum Design MPMS XL5) over a range of temperatures $(2 \mathrm{~K} \leqslant T \leqslant 300 \mathrm{~K})$ and in applied static magnetic fields up to $50 \mathrm{kOe}$. A sample with mass $6.9 \mathrm{mg}$ 
was placed in a plastic capsule that was supported by a plastic sample holder. The diamagnetic contribution of the capsule and holder is insignificant compared with the large magnetic signal of the sample, so no correction was necessary. For $T \leqslant 150 \mathrm{~K}$, the complex ac magnetic susceptibility, $\chi^{\prime}(T, v)+i \chi^{\prime \prime}(T, v)$, was recorded by the same instrument using an ac magnetic field of $2.5 \mathrm{Oe}$ in the frequency interval $1 \mathrm{~Hz} \leqslant v \leqslant 1 \mathrm{kHz}$ while no dc magnetic field was applied.

\section{RESULTS AND DISCUSSION}

\section{A. Structural properties}

Typical micrographs, showing the size and shape of the resulting CoO-Pt core-shell nanoparticles, are shown in Fig. 1, and particle size (log-normal) distribution analysis yielded diameters of $(4.0 \pm 0.2) \mathrm{nm}$. Although HRTEM evidence of the core-shell morphology was not resolved, this result is consistent with the findings of Park and Cheon [45], whose HRTEM images showed only smooth and homogeneous boundaries for solid solutions and core-shell CoPt nanoparticle samples. However, the core-shell nature of our $\mathrm{CoO}-\mathrm{Pt}$ nanoparticles was resolved by our EXAFS experiments, vide infra. Electron diffraction patterns, shown as insets in Fig. 1, clearly indicate the crystalline character of the sample and long-range structural order.

The XRD pattern of our CoO-Pt core-shell nanoparticles is shown in Fig. 2, where the relatively broad Bragg peaks indicate the nanocrystalline nature of the sample. While the analysis suggests the presence of the fcc Pt phase (PDF 40-802), the $\mathrm{CoO}$ phase cannot be resolved unambiguously due to overlapping of the anticipated $\mathrm{CoO}$ peaks with the ones associated with the Pt phase. These observations are typical for core-shell nanostructures of iron or cobalt fine nanoparticles coated with $\mathrm{Au}$ or $\mathrm{Pt}[46,47]$. So, the $\mathrm{CoO}$ phase in our particles was resolved by our EXAFS and XANES studies, vide infra. Nevertheless, the XRD can be used to extract the average grain size from the line broadening by applying the Scherrer formula [48]. More specifically, Bragg peaks at (111), (200), and (220) were fitted to Gaussian functions to extract values for the full width at half maximum (FWHM), which were corrected for the instrumental broadening. Finally, the analysis yielded values for the average size of nanoparticles, $(3.4 \pm 0.4) \mathrm{nm}$, and the lattice parameter, $(3.908 \pm 0.004) \AA$.

In order to provide experimental evidence for the CoO-Pt core-shell morphology of our samples, XAS measurements at the Pt $L_{3}$ and Co $K$ edges were performed. A great advantage of XAS is its chemical sensitivity that provides a microscopic probe of the atomic neighborhood of a selected atomic constituent. Generally, a signal obtained from XAS consists of two parts: (i) the part near the absorption edge, literally named XANES (x-ray-absorption near-edge structure) and (ii) EXAFS (extended x-ray-absorption fine structure) that starts 30-60 eV above an absorption edge and persists up to about $1 \mathrm{keV}$ beyond the edge by revealing specific oscillations of the absorption coefficient. In other words, XANES can provide information about the electronic configuration and is sensitive to the oxidation state of the absorbing atom, while EXAFS contains information about the coordination environment of the absorbing atom.
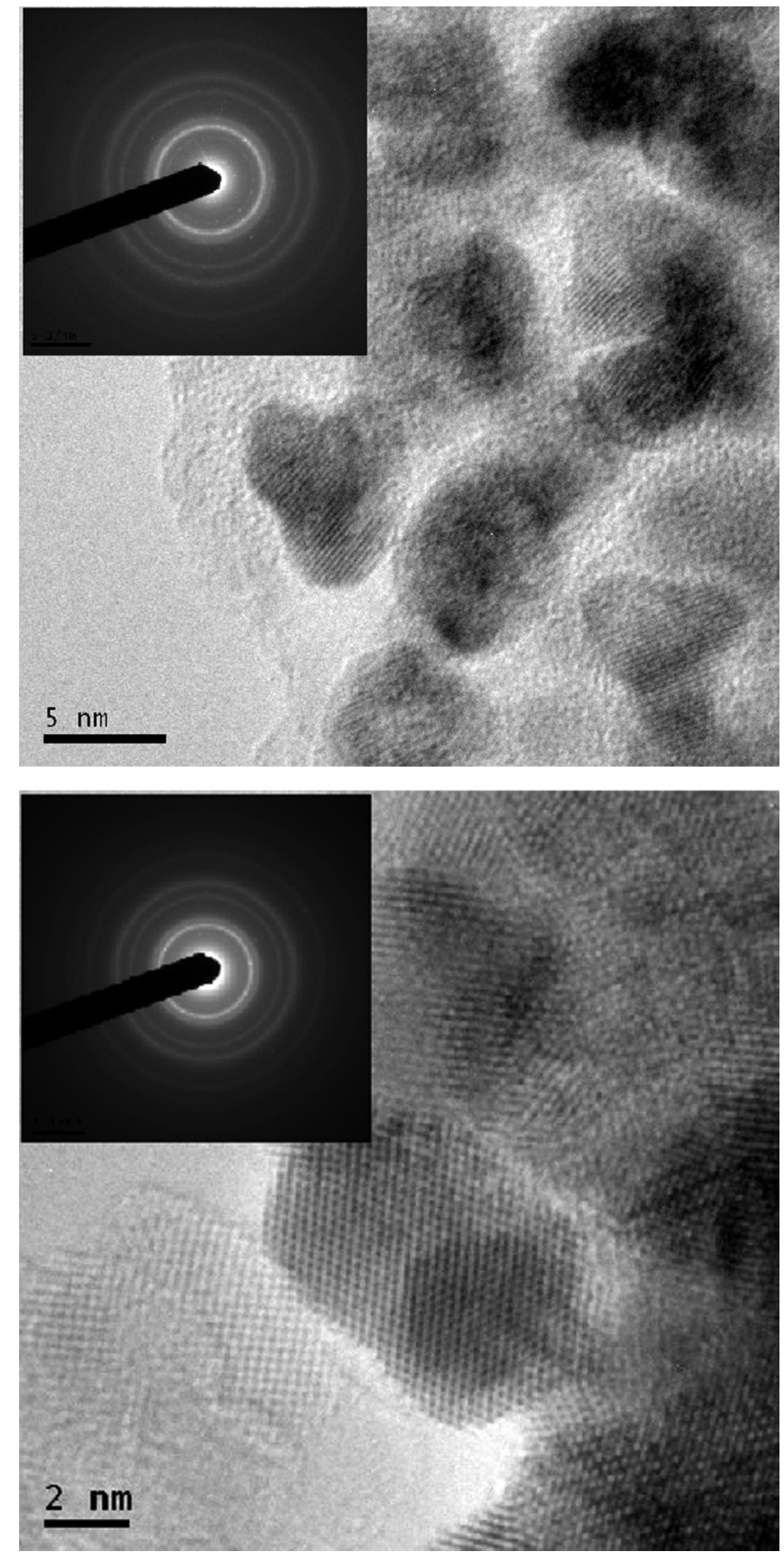

FIG. 1. HRTEM micrographs and diffraction patterns of from two different samples of the CoO-Pt core-shell nanoparticles.

The XANES spectra for nanoparticles obtained at the Pt $L_{3}$ and Co $K$ edges are shown Fig. 3, together with XANES spectra obtained from pure $\mathrm{Pt}$ and Co reference foils (with micrometer size), respectively. In the case of the $\mathrm{Pt} L_{3}$ edge, the XANES signal coming from CoO-Pt core-shell nanoparticles is practically identical to the signal from $\mathrm{Pt}$ reference foil. This behavior indicates that the Pt atoms in the nanoparticles have comparable electronic configurations and the same oxidation states (exactly the same position and the shape of the absorption edge) as Pt atoms in the reference foil. The situation at the Co $K$ edge is completely different, and the XANES signal of CoO-Pt core-shell particles has no common features with XANES extracted from the Co reference foil, as 


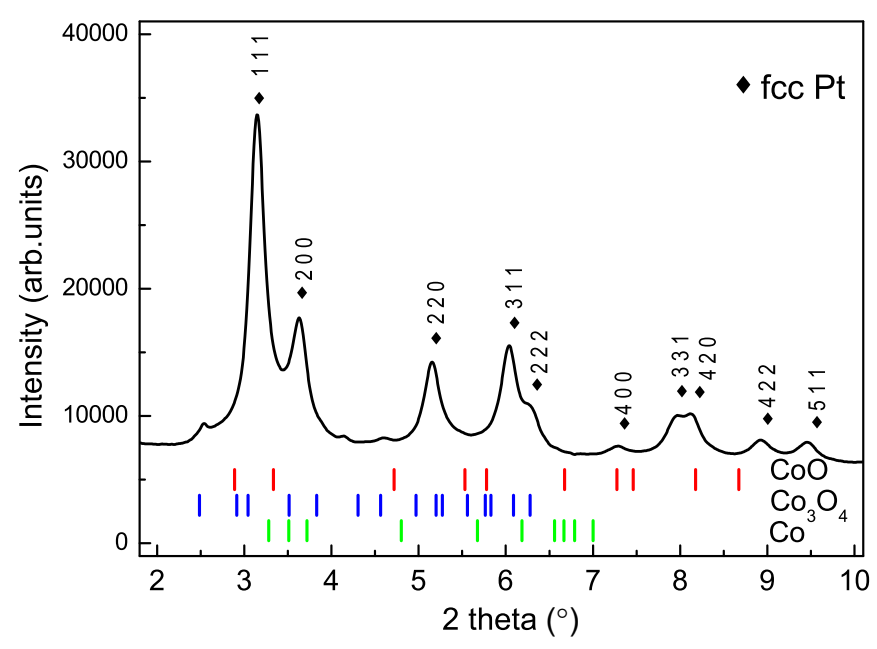

FIG. 2. (Color online) XRD pattern, of CoO-Pt core-shell nanoparticles, obtained using synchrotron radiation with a wavelength $0.12398 \AA$. Peak positions associated with fcc $\mathrm{Pt}, \mathrm{CoO}, \mathrm{Co}_{3} \mathrm{O}_{4}$, and $\mathrm{Co}$ are designated, and a full discussion of the results is given in the text.
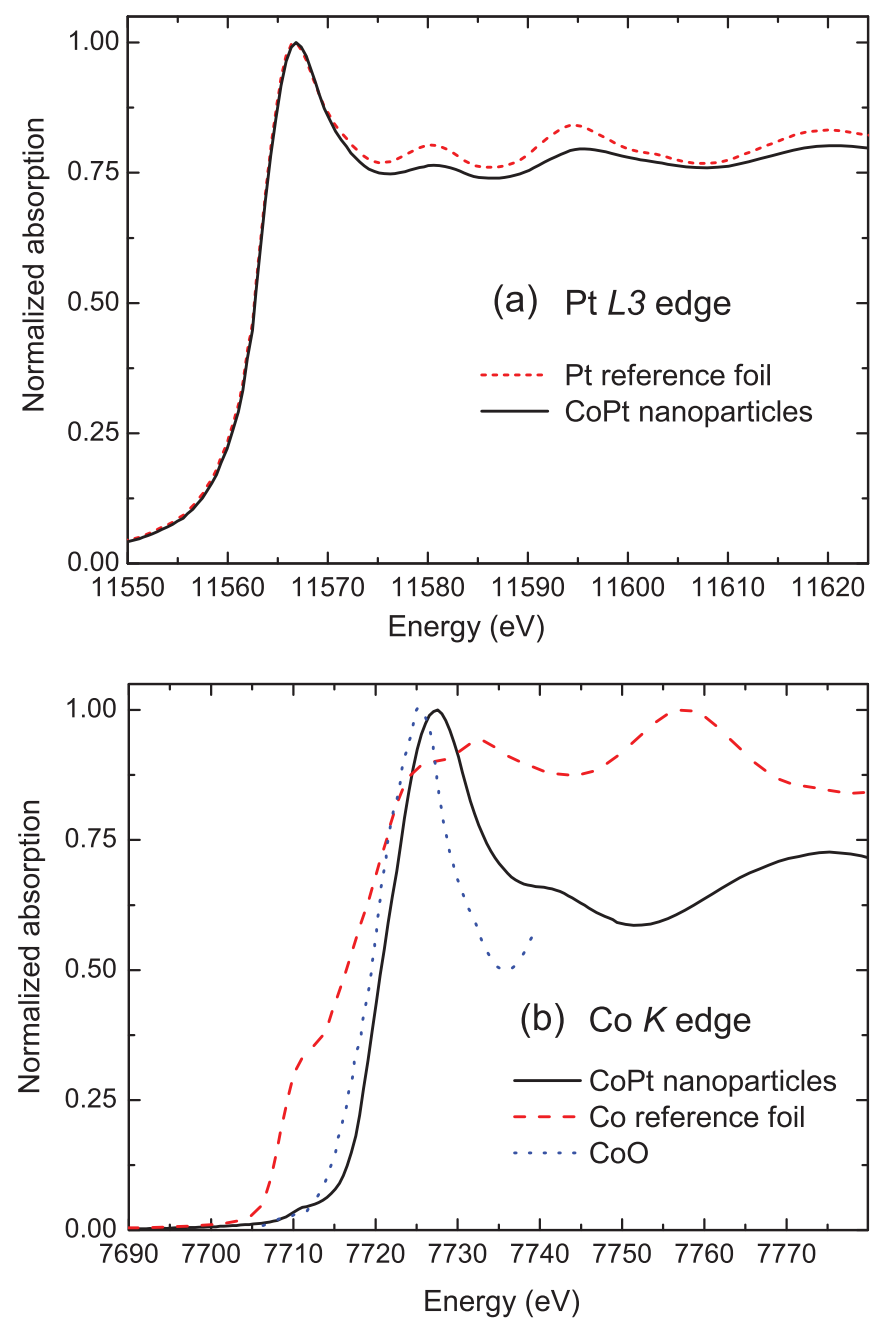

FIG. 3. (Color online) Normalized (a) Pt $L_{3}$ and (b) Co $K$ absorption edges of CoO-Pt core-shell nanoparticles (solid line) and $\mathrm{Pt}$ and Co reference foil (dash line), respectively. Dotted line represents the $\mathrm{CoO}$ reference [49]. documented in Fig. 3(b). This result implies that the Co atoms in the nanoparticles feel a more diverse electronic surrounding than the ones in the reference foil. Shifting and mainly shape changing of the absorption edge suggests a different oxidation state of cobalt atoms, specifically a $\mathrm{CoO}$ phase. These results observed at the Co absorption edge are in accordance with the observations of Cheng and co-authors [49], who used XANES and EXAFS methods for a detailed structural study of Co nanoparticles with different sizes $(3,5$, and $12 \mathrm{~nm})$ prepared under anaerobic conditions. Their XANES results showed that the pattern from the 12-nm Co particles closely resembled the data from the Co foil at the 7712-eV edge, while the pattern for the 5-nm particles had a much smaller shoulder and the 3-nm particles showed almost no signature. In fact, the data [49] from the 3-nm particles are closest to signatures expected from a $\mathrm{CoO}$ standard. In other words, the smallest Co particles $(3 \mathrm{~nm})$ possess a surface layer of oxygen even though the synthesis was realized in anaerobic conditions [49]. The results from our study are consistent with this trend, where the presence of $\mathrm{CoO}$ was identified in the XANES data.

Additional insight about the structure of our samples was obtained from the EXAFS data, shown in Figs. 4 and 5. Immediately obvious is that the Fourier transformation of the weighted signal at the Co $K$ edge is characterized by two well separated peaks situated at 1.6 and $2.7 \AA$; see Fig. 4(b). These peak positions correspond roughly to the most probable interatomic distances between an absorbing atom and its nearest-neighboring atoms. It is important to note that the interatomic distances extracted in this manner are usually underestimated, so one has to apply phase-shift corrections to obtain reasonable values. Nevertheless, the location of the first peak at relatively low $r$ values suggests the presence of atoms, with a small atomic radius, surrounding the Co. Consequently, an inverse Fourier transformation of the first peak can be performed, as described in the preceding section, with $0.61 \AA \leqslant r \leqslant 2.22 \AA$, and fitted by a shell of oxygen atoms. The results of the fit are compared to the data in Fig. 4(a), and the values of the fitting parameters are $N_{\mathrm{Co}-\mathrm{O}}=6.6 \pm 1$, $r_{\mathrm{Co}-\mathrm{O}}=2.05 \pm 0.03 \AA, \sigma^{2}=0.0105 \pm 0.0005 \AA^{2}$ with $R=$ 0.0460 . The same procedure was applied to the second peak, which contains information about the second coordination shell, where the analysis is restricted to $2.19 \AA \leqslant r \leqslant 3.47 \AA$.

To unambiguously confirm that the external shell of nanoparticles consists exclusively of Pt atoms and does not contain any $\mathrm{Co}$ atoms, the $\mathrm{Pt} L_{3}$ EXAFS signal, Fig. 5, was analyzed using two different models. The initial fitting was performed for a cluster of $12 \mathrm{Pt}$ atoms (the first coordination shell) surrounding an absorbing Pt atom with positions corresponding to the atomic positions in fcc $\mathrm{Pt}$ phase, as motivated by the XRD results. The second model is a modification of the first one, but with 4 of the $12 \mathrm{Pt}$ atoms replaced by $\mathrm{Co}$ atoms. This model represents the $\mathrm{Pm} 3 \mathrm{~m}$ $\mathrm{CoPt}_{3}$ phase (PDF 299-499). We have found that including Co atoms in the first coordination shell strongly decreases the fit quality if the coordination number $N_{\mathrm{Pt}-\mathrm{Co}}$ is constrained to the values between 2 and 4 . When leaving $N_{\mathrm{Pt} \text {-Co }}$ unconstrained, its value tended to zero, meaning that there is essentially no contribution from $\mathrm{Co}$ atoms in the first coordination shell. Therefore, we conclude that the shell surrounding the Co core consists exclusively of Pt atoms. As can be seen from Fig. 5, 

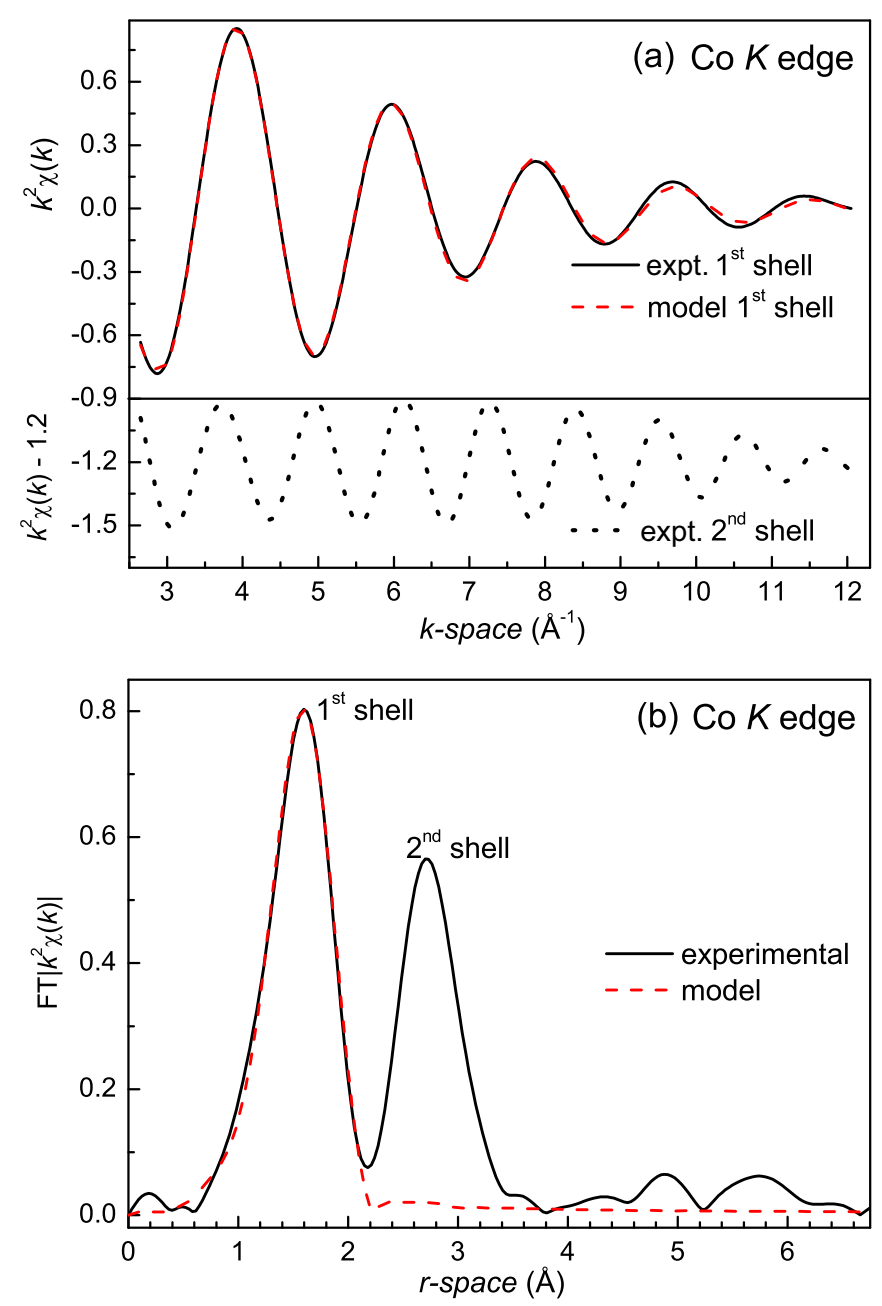

FIG. 4. (Color online) Experimental EXAFS data (solid line) at Co $K$ absorption edge and best-fit result (dash line) for CoO-Pt coreshell nanoparticles. The comparison shows (a) back Fourier transform in $q$ space and (b) magnitude of Fourier transform in $r$ space.

the final fit of the experimental data shows nice agreement with the first model based on a pure fcc Pt phase, where the extracted structural parameters $N_{\mathrm{Pt}-\mathrm{Pt}}=10.3 \pm 1, r_{\mathrm{Pt}-\mathrm{Pt}}=$ $2.73 \pm 0.01 \AA, \sigma^{2}=0.008 \pm 0.0005 \AA^{2}$ with $R=0.0916$. To summarize the structural studies based on results of XRD, XANES, and EXAFS experiments, our samples consist of core $\mathrm{CoO}$ particles with pure Pt shells.

\section{B. dc magnetization}

\section{Temperature dependence}

The temperature dependencies of the zero-field-cooled (ZFC) and field-cooled (FC) dc magnetizations, $M_{\mathrm{ZFC}}$ and $M_{\mathrm{FC}}$, in low static fields $(\leqslant 5000 \mathrm{Oe})$ are shown in Fig. 6. In these low fields, the magnetic response bifurcates at a temperature $T_{\text {irr }}$, exhibiting irreversibility at lower temperatures. In addition, a striking feature is the presence of two discrete ZFC maxima in 5 Oe, suggesting two blocking or freezing temperatures, $T_{\mathrm{M} 1}$ and $T_{\mathrm{M} 2}$. The field dependencies of these three characteristic temperatures are tabulated in Table I.
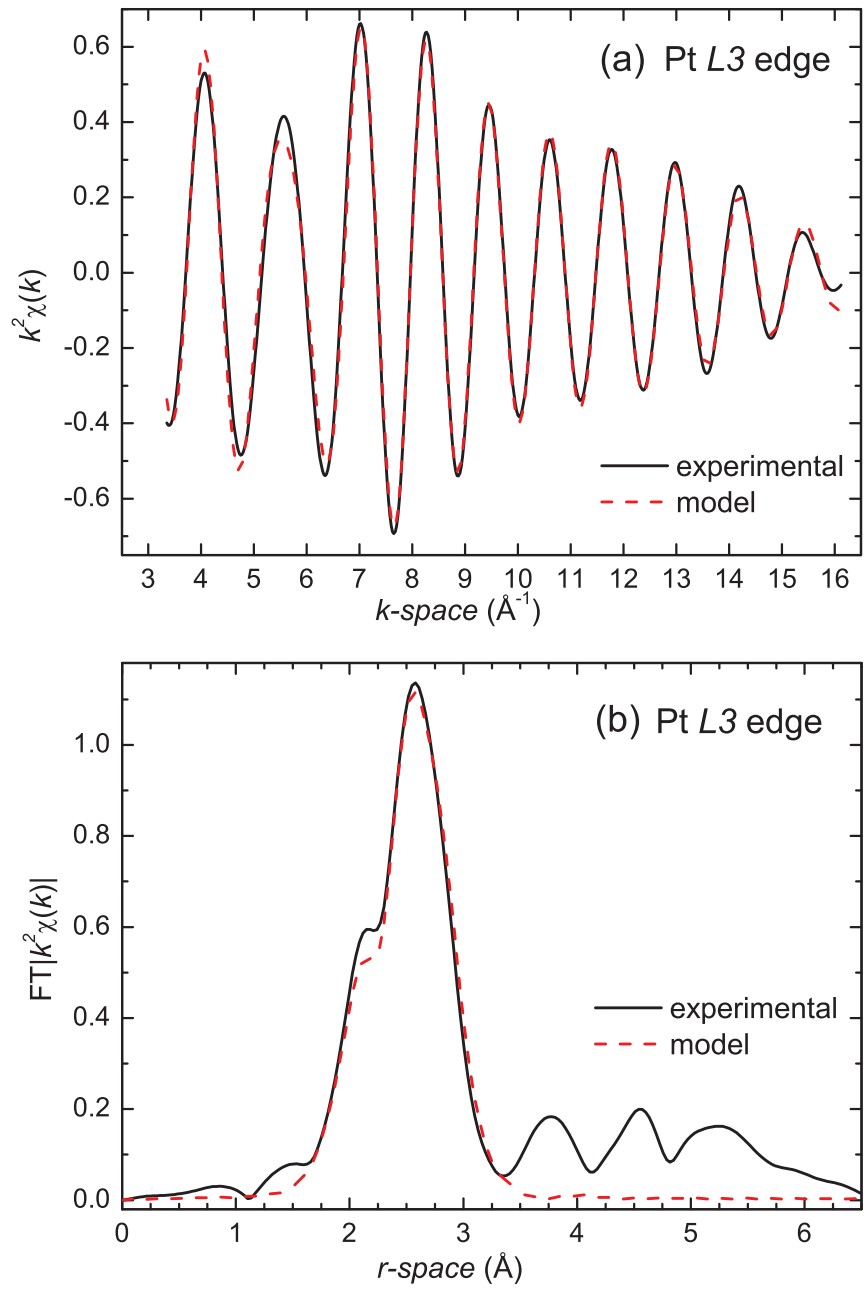

FIG. 5. (Color online) Experimental EXAFS data (solid line) at the $\mathrm{Pt} L_{3}$ absorption edge and best fit result (dash line) for CoO-Pt core-shell nanoparticles. The comparison shows (a) the inverse Fourier transform in $q$ space and (b) the magnitude of Fourier transform in $r$ space.

Another significant feature of the data shown in Fig. 6 is the behavior of $M_{\mathrm{FC}}$, which shows a progressive increase with decreasing temperature below $T_{\mathrm{M} 2}$ and a sharp rise below $T_{\mathrm{M} 1}$. The sharp increase at the lowest temperatures suggests an additional magnetic contribution and may be related to the collective freezing of the disordered spins in the interface "spin" layer between antiferromagnetic $\mathrm{CoO}$ core and nonmagnetic Pt shell.

The presence of two maxima in $M_{\mathrm{ZFC}}(T)$ data acquired in low fields was also reported by Winkler et al. $[15,16]$ and Thota and Kumar [14] for NiO core-shell nanoparticles with antiferromagnetic cores. They associated this peculiar behavior with the freezing of the magnetically ordered region in the surface shell. In addition, Zhang et al. [31] interpreted the anomalous properties of antiferromagnetic $\mathrm{CoO}$ nanoparticles in terms of a core-shell model, where the ferromagnetic portion is attributed to the increase of the uncompensated moments at the surface resulting from the reduced coordination of surface spins. Furthermore, the results of Zysler et al. [13] and Biasi et al. [17] show the important role of the surface anisotropy 


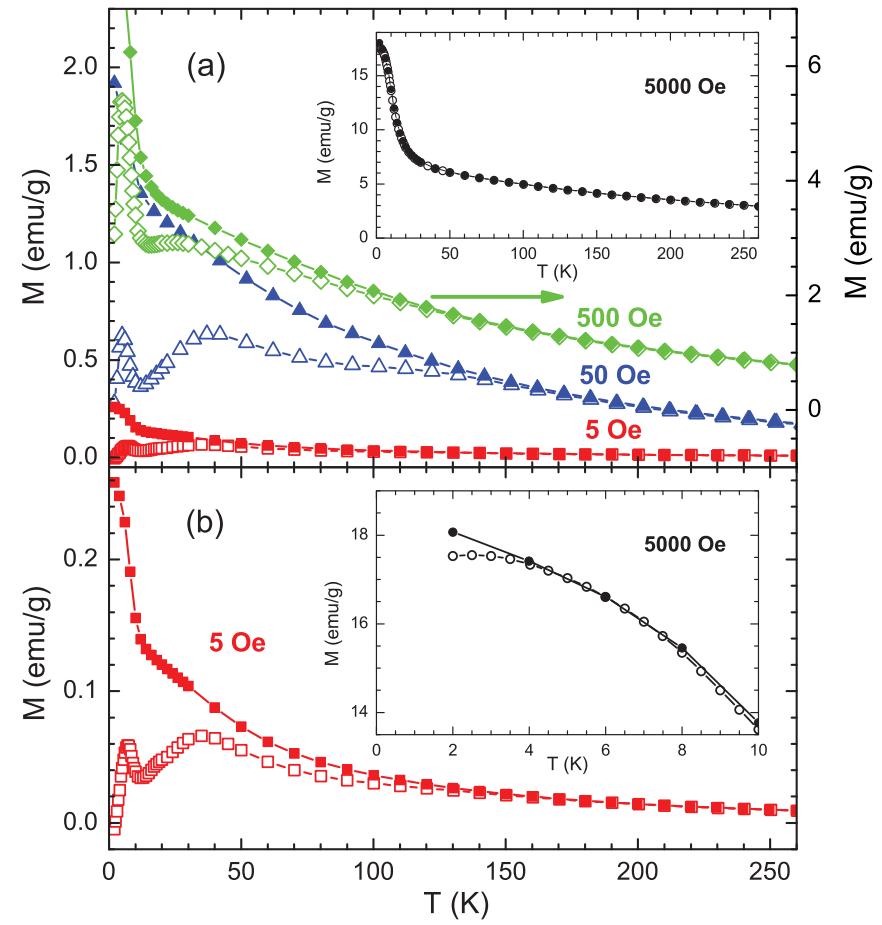

FIG. 6. (Color online) Temperature dependence of the dc magnetization measured in fields up to 5000 Oe after ZFC (open symbols) and FC (solid symbols). The full temperature range is shown in (a), while (b) provides an expanded view of the 5-Oe and 5000-Oe data. Table I provides a tabulation of the features discussed in the text.

in determination of the anomalies of dc magnetization of 3-nm sized magnetic nanoparticles. Likewise, Dutta et al. [50] employed a core-shell model, which assumes the coexistence of an ordered core with disordered surface spins, to explain unconventional magnetic properties of nominally 4-nm ironoxide nanoparticles.

The existence of these two, low-temperature maxima in low magnetic fields begs the question as to the nature of the magnetic state at high temperatures. In an attempt to resolve this issue, magnetic susceptibility $(\chi=M / H)$ times temperature versus temperature plots were constructed, and the results for the data sets in 5 and 5000 Oe are shown in Fig. 7. The data for these two fields show contrasting behavior, as the data in 5 Oe indicate a ferromagnetic trend for $150 \mathrm{~K} \lesssim T \leqslant 300 \mathrm{~K}$ while the results in 5000 Oe indicate an antiferromagnetic trend for nearly all temperatures. In other words, the signatures present in the static magnetic responses appear to possess a complex interplay of temperature and

TABLE I. Field dependencies of the temperatures of the two maxima in the ZFC data and the irreversibility temperatures of the data shown in Fig. 6.

\begin{tabular}{lccc}
\hline \hline $\mathrm{B}(\mathrm{Oe})$ & $T_{\mathrm{M} 1}(\mathrm{~K})$ & $T_{\mathrm{M} 2}(\mathrm{~K})$ & $T_{\mathrm{irr}}(\mathrm{K})$ \\
\hline 5 & 6 & 36 & 168 \\
50 & 5 & 37 & 142 \\
500 & 4 & 29 & 115 \\
5000 & 3 & & 3 \\
\hline \hline
\end{tabular}

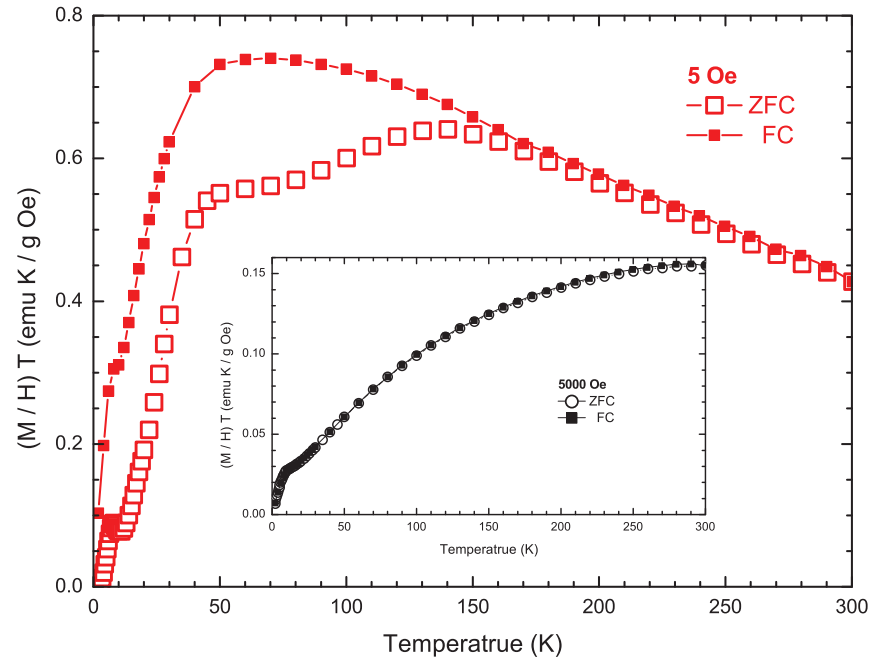

FIG. 7. (Color online) Temperature dependence of the dc magnetization of the CoO-Pt core-shell nanoparticles measured in field 5 Oe when using ZFC and FC protocols. The inset shows $M_{\mathrm{ZFC}}$ and $M_{\mathrm{FC}}$ curves measured in $5000 \mathrm{Oe}$. These data sets in 5 and $5000 \mathrm{Oe}$ show the influence of the magnetic field on the shape of curves.

magnetic field, so the results of isothermal $M(H)$ studies will be presented before a full discussion is presented at the end of this subsection.

\section{Field dependence}

After ZFC, the isothermal $M(H)$ loops were obtained by sweeping to $\pm 50 \mathrm{kOe}$ at several temperatures, Fig. 8. For $T \geqslant 120 \mathrm{~K}$, the magnetization curves are reversible and no coercivity was detected, whereas for $T \leqslant 50 \mathrm{~K}$, hysteresis was observed, and the values of the coercive fields are listed in the legend of Fig. 8(b). For $T \geqslant 50 \mathrm{~K}$, it is noteworthy that a temperature-dependent paramagnetic contribution is observed as a straight line for $30 \mathrm{kOe} \leqslant H \leqslant 50 \mathrm{kOe}$. This observation suggests a modified Langevin formalism, which has been applied to other systems for $T_{\mathrm{B}} \lesssim T \lesssim T_{\mathrm{N}}$ [51-54], might be applied to the $M(H)$ data sets at 50 and $120 \mathrm{~K}$, because neither a classical Langevin function [55] nor a weighted sum of Langevin functions $[8,56]$ plausibly simulated the $M(H)$ data. The modified Langevin analysis provides coarse estimates of the nanoparticle magnetic moments of $1500 \mu_{\mathrm{B}}$ at $50 \mathrm{~K}$ and $2000 \mu_{\mathrm{B}}$ at $120 \mathrm{~K}$, where, presumably, the difference between these two values arises from thermal variations of the antiferromagnetic interactions.

Using the data reported by Silva et al. [32], the moments at 50 and $120 \mathrm{~K}$ might be expected from 4-nm diameter nanoparticles of antiferromagnetically ordered $\mathrm{CoO}$. However, the Néel temperature of nanoparticles of this size is expected to be significantly less than $240 \mathrm{~K} \mathrm{[32].} \mathrm{So,} \mathrm{the} \mathrm{temperature}$ of the maximum value of $\partial(M / H) T / \partial T$, for the ZFC data set with subsequent measuring in 5 Oe, Fig. 8, is near $145 \mathrm{~K}$ and provides an estimate of $T_{\mathrm{N}}[53,57,58]$. In addition, $T_{\mathrm{N}} \approx 148 \mathrm{~K}$ was estimated for a Curie-Weiss law analysis of the data obtained at $10 \mathrm{kOe}$. Also, various analyses of the data consistently indicate $T_{\mathrm{N}}=146 \pm 2 \mathrm{~K}$. 

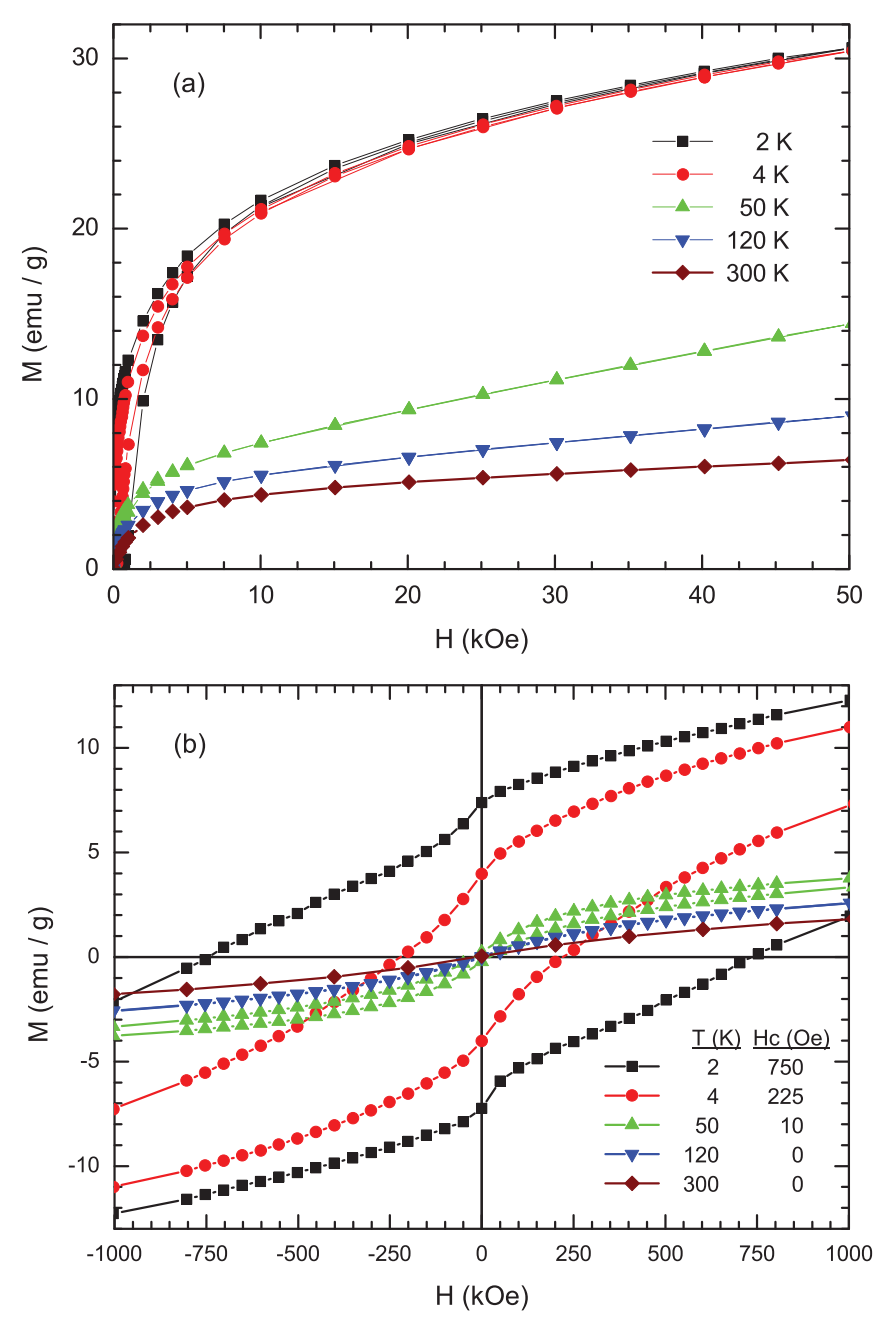

FIG. 8. (Color online) Field dependencies of the isothermal magnetization measured at several different temperatures. The first $M$ vs $H$ quadrant is shown in (a). An expanded view of the low-field region of the hysteresis loops is provided in (b), and the values of the coercive fields are given in the legend.

\section{C. ac susceptibility}

The real (in phase), $\chi^{\prime}(T, v)$, and the imaginary (out of phase), $\chi^{\prime \prime}(T, v)$, magnetic susceptibility are shown in Figs. 9 and 10 after ZFC. The existence of two maxima are observed in both components near the temperatures of the peaks resolved in the dc study, Fig. 6 and Table I, but the ac data reveal these features to be frequency dependent. More specifically, the real part of the magnetic responses shifts toward higher temperatures for both maxima with increasing frequency, while the amplitudes of the magnetic signals decrease. These trends are also reflected in the imaginary part of the magnetic responses although the amplitudes of the magnetic signals show a weak increase. Generally speaking, these results for the dynamical response are characteristics of blocking or freezing processes, so additional inspection of the the thermal and frequency responses are necessary to clarify the nature of the peaks.

The narrow low-temperature peak located at $6 \mathrm{~K}$ exhibits the presence of a "cusplike" maximum in $\chi^{\prime}(T)$, which weakly depends on the frequency. Such characteristics are

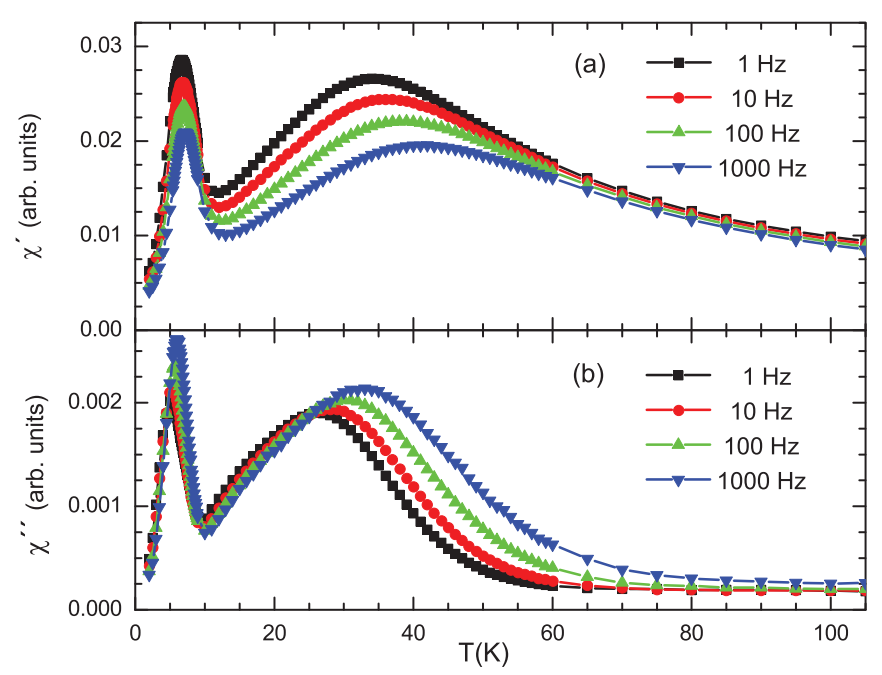

FIG. 9. (Color online) Temperature dependence (a) of in-phase $\chi^{\prime}(T)$ and (b) out-of-phase $\chi^{\prime \prime}(T)$ ac susceptibility at different frequencies of ac field. The data were taken at 1, 10, 100, and $1000 \mathrm{~Hz}$ as indicated in the figure. The first maximum is located at $6 \mathrm{~K}$ while the second maximum occurs at $37 \mathrm{~K}$.

expected for "ideal" spin glasses [19,59]. Contrastingly, the high-temperature peak of $\chi^{\prime}(T)$ located near $37 \mathrm{~K}$ exhibits features associated with a broad distribution and possesses a strong frequency dependence. A useful and sensitive criterion to distinguish between the freezing and the blocking processes lies in determining the relative shift of the peak temperature in $\chi^{\prime}(T)$ given by [59]

$$
p=\frac{\Delta T_{\max }}{T_{\max } \Delta \log v},
$$
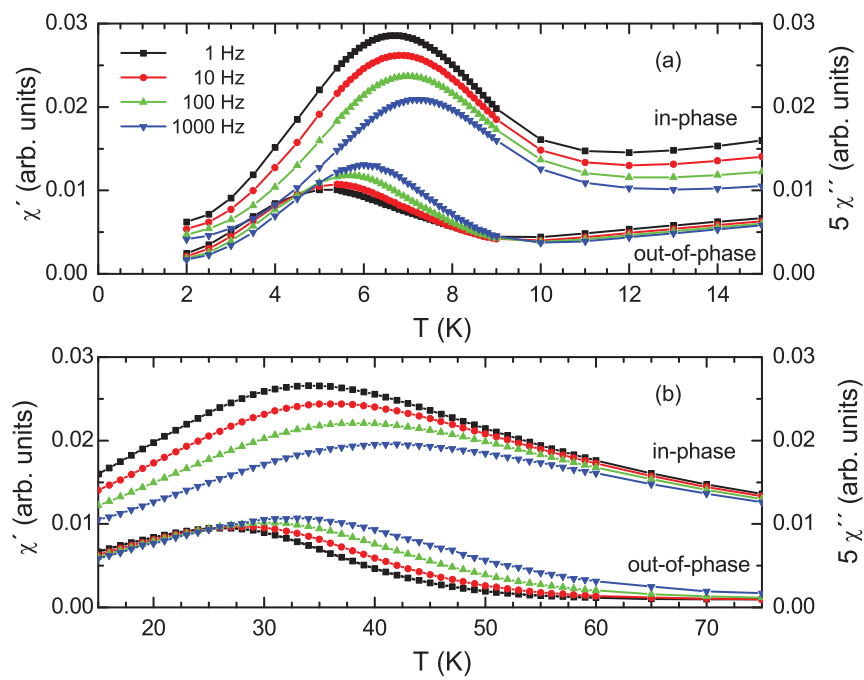

FIG. 10. (Color online) Detail of the (a) low-temperature (sharp) and (b) high-temperature (broad) maxima in the temperature dependence of ac susceptibility. The in-phase $\chi^{\prime}(T)$ and out-of-phase $\chi^{\prime \prime}(T)$ susceptibilities are plotted with the same scale in arbitrary units (arb. units) but are offset for more pronounced comparison with the $\chi^{\prime \prime}(T)$ scale amplified by a factor of 5 . 
where $T_{\max }$ is the average value of the frequency-dependent blocking/freezing temperature determined by the maximum of $\chi^{\prime}(T)$, while $\Delta T_{\max }$ denotes the difference between $T_{\max }$ measured in the $\Delta \log v$ frequency interval. The parameter $p$ assumes values in the range $0.0045-0.06$ for atomic spin glasses $[24,59,60]$ and $0.10-0.13$ for noninteracting superparamagnets $[15,19,59,61]$. These ranges can be compared to the values of $p$ obtained for low-temperature and high-temperature features observed in our CoO-Pt core-shell samples, where $p=0.026$ calculated for the low-temperature maximum falls within the interval typically associated with a spin-glass state. On the other hand, the value of $p=0.08$ calculated for the high-temperature maximum falls below the range usually associated with a noninteracting nanoparticle system $[15,19,59,60]$, and thus the presence of interparticle interactions can be inferred. Now that these qualitative associations have been made, quantitative analysis of the magnetic data will allow additional interpretations to be made.

\section{High-temperature (broad) peak}

To quantitatively analyze the high-temperature peak, the Stoner-Wohlfarth-Néel description can be used [8,62]. In this description, an anisotropy energy barrier $E_{\mathrm{A}}$ blocks the magnetic moments until a sufficient thermal activation energy $k_{\mathrm{B}} T$ relaxes spins from the blocked state to the superparamagnetic state. The dynamics of the noninteracting superparamagnets are described by Néel-Arrhenius law [19,24], which can be written as

$$
\tau=\tau_{0} \exp \left(\frac{E_{\mathrm{A}}}{k_{\mathrm{B}} T_{\max }}\right),
$$

where $\tau$ is the time associated with particle flips between two energy states, $\tau_{0}$ is an attempt frequency, and $T_{\max }$ is the temperature at which $\chi^{\prime}(T)$ exhibits a maximum $[8,24,63]$. For noninteracting particles, typical values for $\tau_{0}$ are usually within the range $10^{-9}-10^{-12} \mathrm{~s}$. Fitting the experimental data to Eq. (2) yields $\tau_{0}=6 \times 10^{-19} \mathrm{~s}$, which is considerably lower than the values expected for noninteracting particles. So the breath of the analysis can be expanded by using the Vogel-Fulcher law $[8,19,63]$, namely

$$
\tau=\tau_{0} \exp \left(\frac{E_{\mathrm{A}}}{k_{\mathrm{B}}\left(T_{\max }-T_{0}\right)}\right),
$$

where $T_{0}$ accounts for a static interaction field due to the moments of surrounding particles [64]. Fitting the data associated with the high-temperature peak with Eq. (3) yields the reasonable results shown in Fig. 11(a). The resulting values for the parameters are $\tau_{0}=2.4 \times 10^{-11} \mathrm{~s}, E_{\mathrm{A}} / k_{\mathrm{B}}=558 \mathrm{~K}$, and $T_{0}=10.8 \pm 0.5 \mathrm{~K}$. The anisotropy barrier $E_{A}$ is related to the uniaxial anisotropy constant $K_{A}$ through the relation $E_{A}=K_{A} V$, where $V$ is the volume of the nanoparticle. For a particle with diameter $d=4 \mathrm{~nm}, K_{A}=2 \times 10^{6} \mathrm{erg} / \mathrm{cm}^{3}$, which is a reasonable result.

\section{Low-temperature (sharp) peak}

The temperature and frequency response of the ac susceptibility in the vicinity of the low-temperature peak are dramatically different than the behavior observed near the high-temperature peak. Since the data are strikingly similar
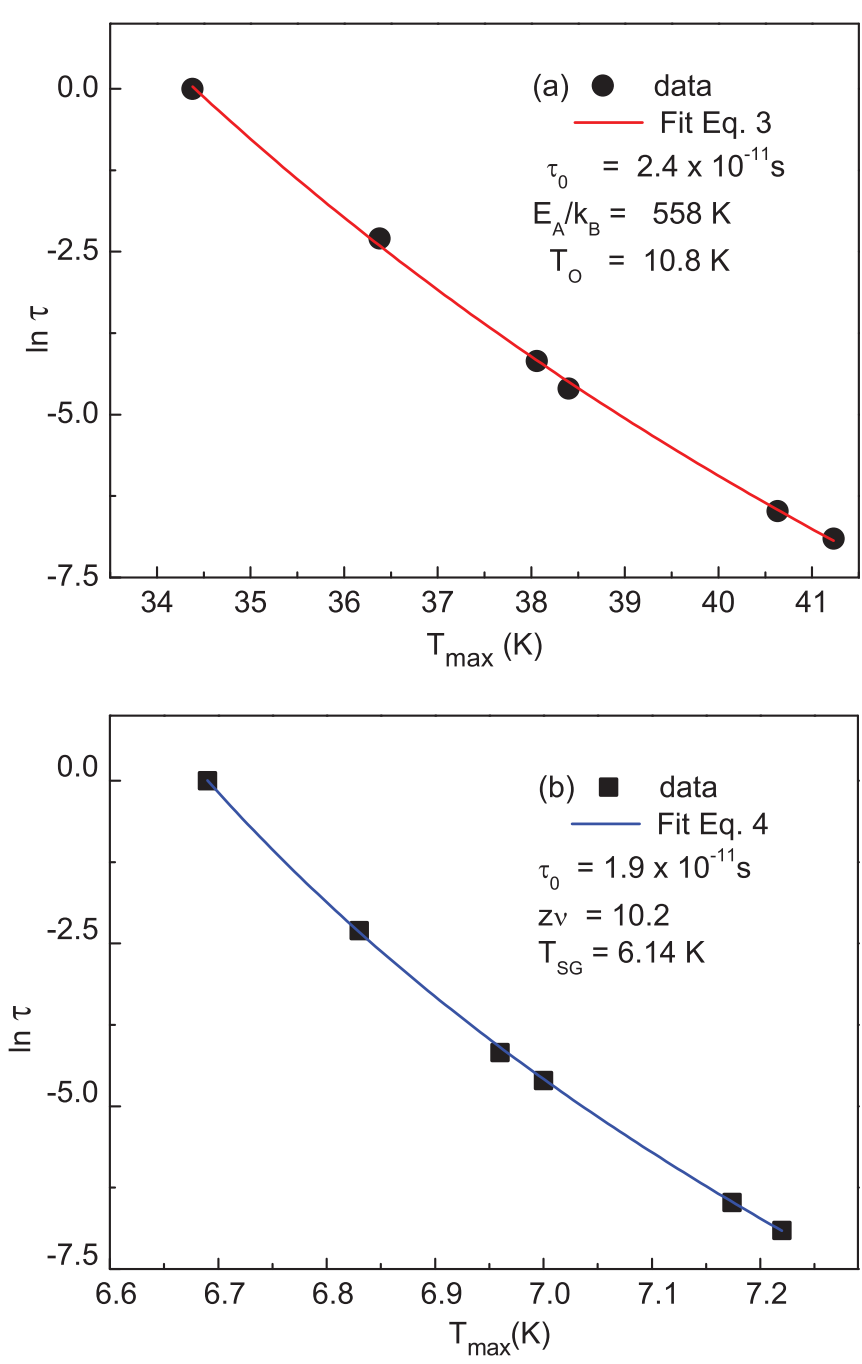

FIG. 11. (Color online) Dependence of $\ln \tau$ vs critical temperature $T_{\mathrm{f}}$ determined from the ac susceptibility at frequencies of 1,10 , 65, 100, 650, and $1000 \mathrm{~Hz}$. In (a), the high-temperature (broad) peak data are fit by Eq. (3), while in (b), the low-temperature (sharp) peak data are fit by Eq. (4); see text for details.

to observations reported in other nanosystems $[62,65,66]$, the analysis begins by differentiating between behavior indicative of superspin glass (SSG) and the atomic spin glass (SG) behavior. Specifically for spin glasses, the spin freezing temperature $T_{\mathrm{f}}(v)$ can be defined as the temperature where the real part of ac susceptibility, $\chi^{\prime}(T, v)$, manifests a maximum [29,67]. Although $T_{\mathrm{f}}$ is often taken as a temperature at which $\chi^{\prime}(T, v)$ is 0.98 times the equilibrium susceptibility, it is reasonable to define $T_{f}(v)$ as a temperature of maximum susceptibility in the $\chi^{\prime}(T, v)$ curve for dynamical scaling analysis, as was demonstrated by Gunnarsson et al. [68] and Djurberg et al. [66]. The dynamic scaling hypothesis provided that this system exhibits a conventional critical slowing down toward the transition temperature $T_{\mathrm{SG}}$, the variation of maximum relaxation time with transition temperature is described by $[19,29,66,69]$

$$
\tau=\tau_{0}\left(\frac{T_{\nu}(v)-T_{\mathrm{SG}}}{T_{\mathrm{SG}}}\right)^{-z v} .
$$


Here, $\tau_{0}$ is the characteristic time scale for the spin dynamics, $T_{\mathrm{SG}}$ is the critical temperature for spin-glass ordering (this is equivalent to the $v \rightarrow 0$ value of $T_{f}$ ), $z v$ is a constant exponent, where $z$ is a dynamic exponent, and $v$ is the critical exponent characterizing the divergence of the correlation length $\xi$ given by [66]

$$
\xi=\left(\frac{T_{\mathrm{SG}}}{\left(T_{\mathrm{SG}}-T_{v}\right)}\right)^{v} .
$$

The agreement of experimental data with Eq. (4) is shown in Fig. 11(b), where the best fit yields the values of $z v=$ $10.2 \pm 0.6, T_{\mathrm{SG}}=6.14 \pm 0.04 \mathrm{~K}$, and $\tau_{0}=1.9 \times 10^{-11} \mathrm{~s}$. These results are comparable with other atomic spin-glass systems [9,12,31,32] and nanoparticle superspin glass systems, where typical values for the parameters are $z v \sim 10$, $\tau_{0} \sim 10^{-11}-10^{-13} \mathrm{~s}$. Together, these results indicate the slow spin dynamics in the vicinity of the low-temperature peak.

\section{SUMMARY}

The structure and the magnetic properties of fine nanoparticles composed of antiferromagnetic $\mathrm{CoO}$ cores coated by a Pt shell, prepared by a reverse micelle method, are presented. A suite of experimental probes were used to establish the structural and magnetic properties of the nanoparticles that possess a nominal diameter of $4 \mathrm{~nm}$. Below about $6 \mathrm{~K}$, the core-shell nanoparticles possess superspin-glass properties.
At higher temperatures, up to about $37 \mathrm{~K}$, the magnetism of the cores is blocked and interparticle interactions play an important role in the dynamical magnetic response. This co-existence of blocking and freezing behaviors is consistent with the nanoparticles possessing an antiferromagnetically ordered core, with an uncompensated magnetic moment, and a magnetically disordered interlayer between $\mathrm{CoO}$ core and $\mathrm{Pt}$ shell. Finally, due to their small diameters and the presence of the Pt shell, the cores experience magnetic ordering near $150 \mathrm{~K}$. Ultimately, these results provide benchmarks by which this system can be judged for potential use in microelectronic or biotechnology applications.

\section{ACKNOWLEDGMENTS}

This work was supported by the Slovak Research and Development Agency under Contracts No. APVV-0222-10 and No. APVV-0132-11 and VEGA projects of Ministry of Education of the Slovak Republic (Grants No. 1/0583/11 and No. 1/0861/12), by the Ministry of Education of the Czech Republic via ECOP program n. cz.1.07/2.3.00/30.0057 (Š.M.), by the US National Science Foundation (NSF) via DMR-1202033 (M.W.M.) and DMR-1157490 (NHMFL), and by the Fulbright Commission of the Slovak Republic (M.W.M.). The authors (A.Z. and V.Z.) would like to thank DESY/HASYLAB, Project No. I-20110282 EC, and also Dr. N. Murafa (AS CR, Rez, Czech Republic) for HRTEM measurements. Computing assistance from V. Tkáč is also gratefully acknowledged.
[1] S. Guo, S. Zhang, L. Wu, and S. Sun, Angew. Chem. Int. Ed. 51, 11770 (2012).

[2] M. Zeisberger, S. Dutz, R. Müller, R. Hergt, N. Matoussevitch, and H. Bönnemann, J. Magn. Magn. Mater. 311, 224 (2007).

[3] C. Osorio-Cantillo, A. N. Santiago-Miranda, O. Perales-Perez, and Y. Xin, J. Appl. Phys. 111, 07B324 (2012).

[4] Y. Kobayashi, M. Horie, M. Konno, B. Rodríguez-González, and L. M. Liz-Marzán, J. Phys. Chem. B 107, 7420 (2003).

[5] R. Morales, Z.-P. Li, J. Olamit, K. Liu, J. M. Alameda, and I. K. Schuller, Phys. Rev. Lett. 102, 097201 (2009).

[6] S. Chandra, H. Khurshid, W. Li, G. C. Hadjipanayis, M. H. Phan, and H. Srikanth, Phys. Rev. B 86, 014426 (2012).

[7] P. Jönsson, J. L. García-Palacios, M. F. Hansen, and P. Nordblad, J. Mol. Liq. 114, 131 (2004).

[8] M. Tadic, V. Kusigerski, D. Markovic, I. Milosevic, and V. Spasojevic, J. Magn. Magn. Mater. 321, 12 (2009).

[9] P. E. Jönsson and J. L. García-Palacios, Phys. Rev. B 64, 174416 (2001).

[10] J. B. Lee, W. G. Hong, H. J. Kim, Z. Jaglicic, S. Jazbec, M. Wencka, A. Jelen, and J. Dolinsek, Phys. Rev. B 86, 224407 (2012)

[11] S. Sahoo, O. Petracic, C. Binek, W. Kleemann, J. B. Sousa, S. Cardoso, and P. P. Freitas, Phys. Rev. B 65, 134406 (2002).

[12] S. Mørup, M. F. Hansen, and C. Frandsen, Beilstein J. Nanotechnol. 1, 182 (2010).

[13] R. Zysler, H. Romero, C. Ramos, E. Biasi, and D. Fiorani, J. Magn. Magn. Mater. 266, 233 (2003).
[14] S. Thota and J. Kumar, J. Phys. Chem. Solids 68, 1951 (2007).

[15] E. Winkler, R. D. Zysler, M. Vasquez Mansilla, and D. Fiorani, Phys. Rev. B 72, 132409 (2005).

[16] E. Winkler, R. D. Zysler, M. V. Mansilla, D. Fiorani, D. Rinaldi, M. Vasilakaki, and K. N. Trohidou, Nanotechnology 19, 185702 (2008).

[17] E. De Biasi, R. D. Zysler, C. A. Ramos, H. Romero, and D. Fiorani, Phys. Rev. B 71, 104408 (2005).

[18] M. Jamet, M. Ngrier, V. Dupuis, J. Tuaillon-Combes, P. Mlinon, A. Prez, W. Wernsdorfer, B. Barbara, and B. Baguenard, J. Magn. Magn. Mater. 237, 293 (2001).

[19] J. A. De Toro, M. A. López de la Torre, J. M. Riveiro, R. Sáez Puche, A. Gómez-Herrero, and L. C. Otero-Díaz, Phys. Rev. B 60, 12918 (1999).

[20] T. Ambrose and C. L. Chien, Phys. Rev. Lett. 76, 1743 (1996).

[21] A. S. Arrott, Phys. Rev. B 31, 2851 (1985).

[22] M. D. Rechtin, S. C. Moss, and B. L. Averbach, Phys. Rev. Lett. 24, 1485 (1970).

[23] I. Golosovsky, I. Mirebeau, G. Andr, M. Tovar, D. Tobbens, D. Kurdyukov, and Y. Kumzerov, Phys. Solid State 48, 2130 (2006).

[24] S. D. Tiwari and K. P. Rajeev, Phys. Rev. B 72, 104433 (2005).

[25] J. Wang and X. Zeng, in Nanoscale Magnetic Materials and Applications, edited by J. P. Liu, E. Fullerton, O. Gutfleisch, and D. Sellmyer (Springer, New York, 2009), pp. 35-65.

[26] A. Punnoose, H. Magnone, M. S. Seehra, and J. Bonevich, Phys. Rev. B 64, 174420 (2001). 
[27] V. Singh, V. Srinivas, M. Ranot, S. Angappane, and J.-G. Park, Phys. Rev. B 82, 054417 (2010).

[28] J. D. Rall and M. S. Seehra, J. Phys.: Condens. Matter 24, 076002 (2012).

[29] H. Akamatsu, K. Tanaka, K. Fujita, and S. Murai, Phys. Rev. B 74, 012411 (2006).

[30] A. Punnoose, M. Seehra, J. van Tol, and L. Brunel, J. Magn. Magn. Mater. 288, 168 (2005).

[31] L. Zhang, D. Xue, and C. Gao, J. Magn. Magn. Mater. 267, 111 (2003).

[32] N. J. O. Silva, A. Millán, F. Palacio, M. Martins, T. Trindade, I. Puente-Orench, and J. Campo, Phys. Rev. B 82, 094433 (2010).

[33] S. Gubin and Y. Koksharov, Inorg. Mater. 38, 1085 (2002).

[34] N. C. Tombs and H. P. Rooksby, Nature (London) 165, 442 (1950).

[35] M. D. Rechtin and B. L. Averbach, Phys. Rev. Lett. 26, 1483 (1971).

[36] P. S. Silinsky and M. S. Seehra, Phys. Rev. B 24, 419 (1981).

[37] E. Carpenter, C. Sangregorio, and C. O'Connor, IEEE Trans. Magn. 35, 3496 (1999).

[38] J. Lin, W. Zhou, A. Kumbhar, J. Wiemann, J. Fang, E. Carpenter, and C. O'Connor, J. Solid State Chem. 159, 26 (2001).

[39] J.-K. Lee and S.-M. Choi, Bull. Korean Chem. Soc. 24, 32 (2003).

[40] A. P. Hammersley, S. O. Svensson, M. Hanfland, A. N. Fitch, and D. Hausermann, High Press. Res. 14, 235 (1996).

[41] K. V. Klementev, J. Phys. D: Appl. Phys. 34, 209 (2001).

[42] K. Saksl, J. Bednark, R. Nicula, E. Burkel, S. Roth, and H. Franz, J. Phys.: Condens. Matter 19, 176215 (2007).

[43] K. V. Klementiev, VIPER: Visual Processing in EXAFS Researches (2013), http://www.cells.es/Beamlines/CLAESS/ software/viper.html

[44] A. L. Ankudinov, B. Ravel, J. J. Rehr, and S. D. Conradson, Phys. Rev. B 58, 7565 (1998).

[45] J.-I. Park and J. Cheon, J. Am. Chem. Soc. 123, 5743 (2001).

[46] E. E. Carpenter, J. Magn. Magn. Mater. 225, 17 (2001).

[47] J.-I. Park, M. G. Kim, Y.-w. Jun, J. S. Lee, W.-r. Lee, and J. Cheon, J. Am. Chem. Soc. 126, 9072 (2004).

[48] P. Scherrer, Göttinger Nachrichten Gesell. 2, 98 (1918).

[49] G. Cheng, J. D. Carter, and T. Guo, Chem. Phys. Lett. 400, 122 (2004).
[50] P. Dutta, S. Pal, M. S. Seehra, N. Shah, and G. P. Huffman, J. Appl. Phys. 105, 07B501 (2009).

[51] S. A. Makhlouf, F. T. Parker, and A. E. Berkowitz, Phys. Rev. B 55, R14717 (1997).

[52] M. S. Seehra, V. S. Babu, A. Manivannan, and J. W. Lynn, Phys. Rev. B 61, 3513 (2000).

[53] M. S. Seehra and A. Punnoose, Phys. Rev. B 64, 132410 (2001).

[54] A. Punnoose, T. Phanthavady, M. S. Seehra, N. Shah, and G. P. Huffman, Phys. Rev. B 69, 054425 (2004).

[55] D. Kim, Y. Zhang, W. Voit, K. Rao, and M. Muhammed, J. Magn. Magn. Mater. 225, 30 (2001).

[56] F. C. Fonseca, G. F. Goya, R. F. Jardim, R. Muccillo, N. L. V. Carreño, E. Longo, and E. R. Leite, Phys. Rev. B 66, 104406 (2002).

[57] E. E. Bragg and M. S. Seehra, Phys. Rev. B 7, 4197 (1973).

[58] P. Dutta, M. S. Seehra, S. Thota, and J. Kumar, J. Phys.: Condens. Matter 20, 015218 (2008).

[59] J. A. Mydosh, Spin Glasses: An Experimental Introduction (Taylor \& Francis, London, 1994), pp. 66-67.

[60] G. F. Goya and M. P. Morales, J. Meta. Nanocryst. Mater. 20-21, 673 (2004).

[61] D. Peddis, D. Rinaldi, G. Ennas, A. Scano, E. Agostinelli, and D. Fiorani, Phys. Chem. Chem. Phys. 14, 3162 (2012).

[62] C. Vázquez-Vázquez, M. López-Quintela, M. Buján-Núñez, and J. Rivas, J. Nanopart. Res. 13, 1663 (2011).

[63] F. Luis, F. Bartolom, F. Petroff, J. Bartolom, L. M. Garca, C. Deranlot, H. Jaffrs, M. J. Martnez, P. Bencok, F. Wilhelm, A. Rogalev, and N. B. Brookes, Europhys. Lett. 76, 142 (2006).

[64] V. Singh, M. S. Seehra, and J. Bonevich, J. Appl. Phys. 105, 07B518 (2009).

[65] J. A. De Toro, J. A. González, P. S. Normile, P. Muñiz, J. P. Andrés, R. López Antón, J. Canales-Vázquez, and J. M. Riveiro, Phys. Rev. B 85, 054429 (2012).

[66] C. Djurberg, P. Svedlindh, P. Nordblad, M. F. Hansen, F. Bødker, and S. Mørup, Phys. Rev. Lett. 79, 5154 (1997).

[67] O. Petracic, X. Chen, S. Bedanta, W. Kleemann, S. Sahoo, S. Cardoso, and P. Freitas, J. Magn. Magn. Mater. 300, 192 (2006).

[68] K. Gunnarsson, P. Svedlindh, J.-O. Andersson, P. Nordblad, L. Lundgren, H. Aruga Katori, and A. Ito, Phys. Rev. B 46, 8227 (1992).

[69] J. Wu and C. Leighton, Phys. Rev. B 67, 174408 (2003). 